\title{
A Study on the Role of Cartridge Based Nucleic Acid Amplification Test (CBNAAT) for Diagnosing Pediatric Tuberculosis in a Tertiary Care Hospital in Eastern India
}

\author{
Kumar Anshu, Suman Das* and Dilip Kumar Paul \\ Department of Pediatrics, Dr. Bidhan Chandra Roy Post Graduate Institute of Pediatric Sciences, India
}

Submission: December 13, 2017; Published: February 16, 2018

*Corresponding author: Dr. Suman Das, Residential Medical Officer cum Clinical Tutor at Dr. Bidhan Chandra Roy Post Graduate Institute of Pediatric Sciences, 111 Narkeldanga Main Road, Kolkata - 700054, India, Tel: 91-9836721415; Email: dr.sumands@gmail.com

\begin{abstract}
Introduction: India accounts for one-fifth of the global tuberculosis (TB) incidence, with TB remaining one of the leading causes of childhood mortality and morbidity. Bacteriological confirmation of TB in children is challenging due to difficulty in obtaining quality specimens, in the absence of which diagnosis largely depends on clinical judgement. Lack of high sensitivity tests adds to the diagnostic challenge. This study focuses on finding the sensitivity, specificity, positive predictive value and negative predictive value of CBNAAT for diagnosis of TB in pediatric population in pulmonary and extra pulmonary specimens.

Methodology: This study was carried out at department of Pediatric Medicine, Dr. Bidhan Chandra Roy Post Graduate Institute of Pediatric Sciences, Kolkata from July 2016 to July 2017. Total 397 patients showing symptoms and signs of suspected localized and/or disseminated tuberculosis or having history of close contact with diagnosed tuberculosis patients admitted in our hospital during the study period were included in this study. Samples(pulmonary and extrapulmonary) were collected from the subjects and put to test for CBNAAT, Zeihl-Neelsen (ZN) smear and culture.
\end{abstract}

Results: Among the pulmonary samples, CBNAAT detected MTB in 21 of the 98 sputum/induced sputum samples(21.4\%), 74 of the 224 gastric lavage/aspirate samples(33\%) and 0 of the 3 bronchoalveolar lavage samples(0\%). Among the extrapulmonary samples, CBNAAT detected MTB in 7 of the 55 CSF samples(12.7\%), 1 of the 10 pleural fluid samples(10\%), 0 of the 4 ascitic fluid samples(0\%) and 2 of the 3 lymph node aspirate samples obtained by FNAC(66.7\%). Sensitivity, specificity, positive predictive value and negative predictive value of CBNAAT in reference to culture are $92.7 \%, 98.9 \%, 97.1 \%$ and $97.2 \%$ respectively. Sensitivity, specificity, positive predictive value and negative predictive value of CBNAAT in reference to ZN smear are $100 \%, 90.68 \%, 71.42 \%$ and $100 \%$ respectively. CBNAAT also detected 6 cases of rifampicin resistance among the 105 cases detected by CBNAAT (5.7\%).

Conclusion: CBNAAT assay is a rapid test which identifies both the presence of Mycobacterium tuberculosis (MTB) (with high sensitivity, specificity, positive predictive value and negative predictive value) and rifampicin resistance associated with mutation of rpoB gene in a single test. It also helps to avoid injudicious use of anti-tuberculosis drugs.

Keywords: CBNAAT; MTB; ZN smear; Culture

\section{Introduction}

Tuberculosis (TB), an infectious disease caused by Mycobacterium tuberculosis, has an estimated global annual incidence of 9.6 million with 2.2 million cases in India according to World Health Organization (WHO) Global TB Report (2015) Thus,23\% of global annual TB incidents occur in India making it the highest TB burden country [1]. While, globally the exact burden of childhood TB is not well documented, it is estimated that childhood TB constitutes about $10-20 \%$ of all TB cases, in high burden countries $[2,3]$ and TB remains one of the leading cause of childhood mortality and morbidity [4]. In 2013, 63,919 pediatric TB cases were notified accounting for $5 \%$ of notified TB cases [5] in India, under the Revised National Tuberculosis Control Programme (RNTCP).

Diagnosis of pulmonary TB in children is challenging, more so in resource-limited, tuberculosis-endemic countries 
and is largely based on clinical and radiological findings and medical history [6,7]. Bacteriological confirmation of pulmonary TB is challenging due to difficulty in obtaining good quality sputum specimens from children. In the absence of quality specimens, one has to rely on testing alternative specimens which is challenging due to difficulties in obtaining these specimens, inadequate clinical sample volumes and paucibacillary nature of biological samples[8, 9]. Diagnostic efforts are also undermined by the lack of simple diagnostic tests with high sensitivity that can be applied at the point of clinical care [8]. Isolation of mycobacteria by culture, while considered as gold standard for diagnosing TB, takes 4-8 weeks and often requires expensive and sophisticated laboratory facilities which cannot be afforded in most resource-limited settings [9]. Both antigen and antibody TB ELISA tests are poorly sensitive and specific and are not recommended for diagnosis of tuberculosis [10]. Interferon gamma release assays (IGRAs) are being used in place of the skin test in low prevalence countries to detect latent TB infection. However, these expensive tests do not differentiate the TB infection from disease. Also its exact utility in high burden situation is still not clear [11].

The specificities and sensitivities of the polymerase chain reaction (PCR) based diagnostic tests are quite variable $[8,11]$. Further, these tests involve multiple manual steps and long turnaround time, making them unsuitable for decentralized deployment. A series of meta-analyses have shown cartridge based nucleic acid amplification test (CBNAAT)/ Xpert MTB/ RIF to have a high specificity with variable sensitivity in different type of specimens for TB diagnosis [8,12-14]. In 2013 , the WHO endorsed the use of CBNAAT for TB diagnosis in pediatric presumptive pulmonary and extra-pulmonary tuberculosis (EPTB) cases $[15,16]$. CBNAAT, a tool with a quick turn-around time, which simultaneously detects TB and rifampicin resistance, offers a promising solution to achieve the global objective of improved TB care and control and early TB case detection [17].

We aimed to study the diagnostic usefulness of CBNAAT (sensitivity, specificity, positive predictive value and negative predictive value) for diagnosing pediatric tuberculosis in our hospital setting.

\section{Materials and Methods}

The present study was carried out at department of pediatric medicine, Dr. Bidhan Chandra Roy Post graduate institute of pediatric sciences, Kolkataafter taking institutional ethics committee approval from July 2016 to July 2017. Total 397 patients showing symptoms and signs of suspected localized and/or disseminated tuberculosis or having history of close contact with diagnosed tuberculosis patients admitted in our hospital during the study period were included in this study. The exclusion criteria being parents not giving consent.

\section{Study tools}

- $\quad$ Relevant history and clinical examination.Clinical history regarding current complaints of fever, cough, sputum production, haemoptysis, and weight loss was taken. All patients were evaluated for headache, seizures, chest pain, breathlessness and neck swelling or any other evidence of extrapulmonary tuberculosis. Case definition of TB suspectchildren with persistent fever and/or cough for more than 2 weeks, loss of weight/no weight gain(History of unexplained weight loss or no weight gain in past 3 months; loss of weight is defined as loss of more than $5 \%$ body weight as compared to highest weight recorded in last 3 months) and/or history of contact with infectious TB cases(In a symptomatic child, contact with a person with any form of active TB within last 2 years maybe significant) [1].

Presumptive extrapulmonary TB- presence of organ specific symptoms and signs like swelling of lymph node, pain and swelling in joints, neck stiffness, disorientation, etc and/or constitutional symptoms like significant weight loss, persistent fever for more than 2 weeks, night sweats.

- Laboratory investigations- complete hemograms, erythrocyte sedimentation rate (ESR), Mantoux test, ZeihlNeelsen (ZN) smear, fine needle aspiration cytology (FNAC), histopathology (HPE) of samples, and culture.

- Instruments for radiological studies eg. Chest radiograph, ultrasonography (USG), computed tomography (CT) scan, magnetic resonance imaging (MRI) scan, magnetic resonance spectroscopy (MRS) if indicated.

- CBNAAT of samples [sputum/induced sputum, gastric aspirate/lavage, cerebrospinal fluid (CSF), pleural fluid, ascitic fluid, bronchoalveolar lavage (BAL), FNAC material].

Indication of performing BAL- To get adequate samples for testingin patients with a low bacterial load or in those who do not expectoratewith a clinical and radiological

suspicion of pulmonary tuberculosis [18].

\section{Study technique}

Patients were selected as per the inclusion criteria and recruited in the study. Detailed history taking, physical examination and relevant laboratory investigations were done. A pre designed semi structured proforma was used to obtain data based on socio-economic profile, clinical profile and investigations after explaining the purpose of the study and obtaining informed consent from the parent/guardian of the child in writing.

All the samples were collected in well labelled falcon tubes. In pulmonary cases two sputum/induced sputum (Sputum induction was done in a well-ventilated room with an ultrasonic nebuliser and nebulisation done with $10-20 \mathrm{~mL}$ of $3 \%$ hypertonic saline until patient coughed up at least 2 
$\mathrm{mL}$ of sputum or a maximum of 15 minutes.) samples were collected: one early morning and other supervised spot specimen. Smears of both the sputum samples were made, stained by ZN procedure, examined under light microscope. Thereafter, if the samples were positive for acid fast bacilli (AFB), then the positive sample otherwise good quality or early morning sample was used for CBNAAT. So, only one sample was further processed for CBNAAT. In infants and small children gastric lavage was collected in place of sputum. Where invasive techniques like BAL were required, only one sample was collected.Bronchoscopy was done with Pentax fibre optic bronchoscope (FOB) under local anaesthesia with $4 \%$ lignocaine jelly and mouth spray. Bronchial washing and lavage was performed by instilling $20 \mathrm{~mL}$ aliquots of normal saline at room temperature upto $100-120 \mathrm{~mL}$ and collected into a sterile suction trap by aspiration. In extrapulmonary cases the samples collected were CSF by lumbar puncture (LP), lymph node fluid by FNAC, pleural fluid by pleural tap and ascitic fluid by ascitic tap.

\section{CBNAAT}

CBNAAT samples were sent to State TB Training and Demonstration Centre, Dr. B C Roy Polio Clinic and Hospital for Crippled Children. CBNAAT was performed according to the manufacturer's instructions (CEPHEID, Sunnyvale, CA, USA). Our machine contains 4 cartridges so 4 samples were processed foreach run. According to standard operating procedure the sampling reagent (containing $\mathrm{NaOH}$ and isopropanol) was added at2:1 ratio to the sample and kept for 15 minutes at roomtemperature with intermittent shaking. $3 \mathrm{ml}$ of this treated samplewas transferred to the cartridge and the cartridge was inserted inthe module of CBNAAT machine.
An automatic process completedthe remaining assay steps and the results were displayed on themonitor attached to Gene Xpert after $1 \mathrm{hr}$ and 50 minutes.

\section{AFB Smear}

A minimum of 1 slide positive even for single $\mathrm{AFB} / 100$ fields was taken as positive for Mycobacterium tuberculosisand a minimum of two samples negative for AFB evaluated for 100 fields were declared as negative.

\section{Culture}

The concentrated sample obtained after decontamination was inoculated for culture in BACTEC mycobacterium growth indicator tube (MGIT) for liquid culture. Culture was considered as gold standard for analysis of results. When the tubes were flagged positive by the system, ZN staining and culture on $5 \%$ sheep blood agar were performed from the tube directly to see any contamination as per the manufacturer's instructions. All tubes were checked for positivity till 42 days. Mycobacteria other than tuberculosis (MOTT) and Mycobacterium tuberculosis testing from positive culture tubes were done by rapid immunochromatography test kit using MPT 64 antigen according to the manufacturer's instructions.

\section{Data analysis}

All recorded data were analyzed using standard statistical methods (SPSS software). Sensitivity, specificity, positive predictive value and negative predictive value of CBNAAT were calculated. Values of $\mathrm{P}<0.05$ were considered as statistically significant.

\section{Results}

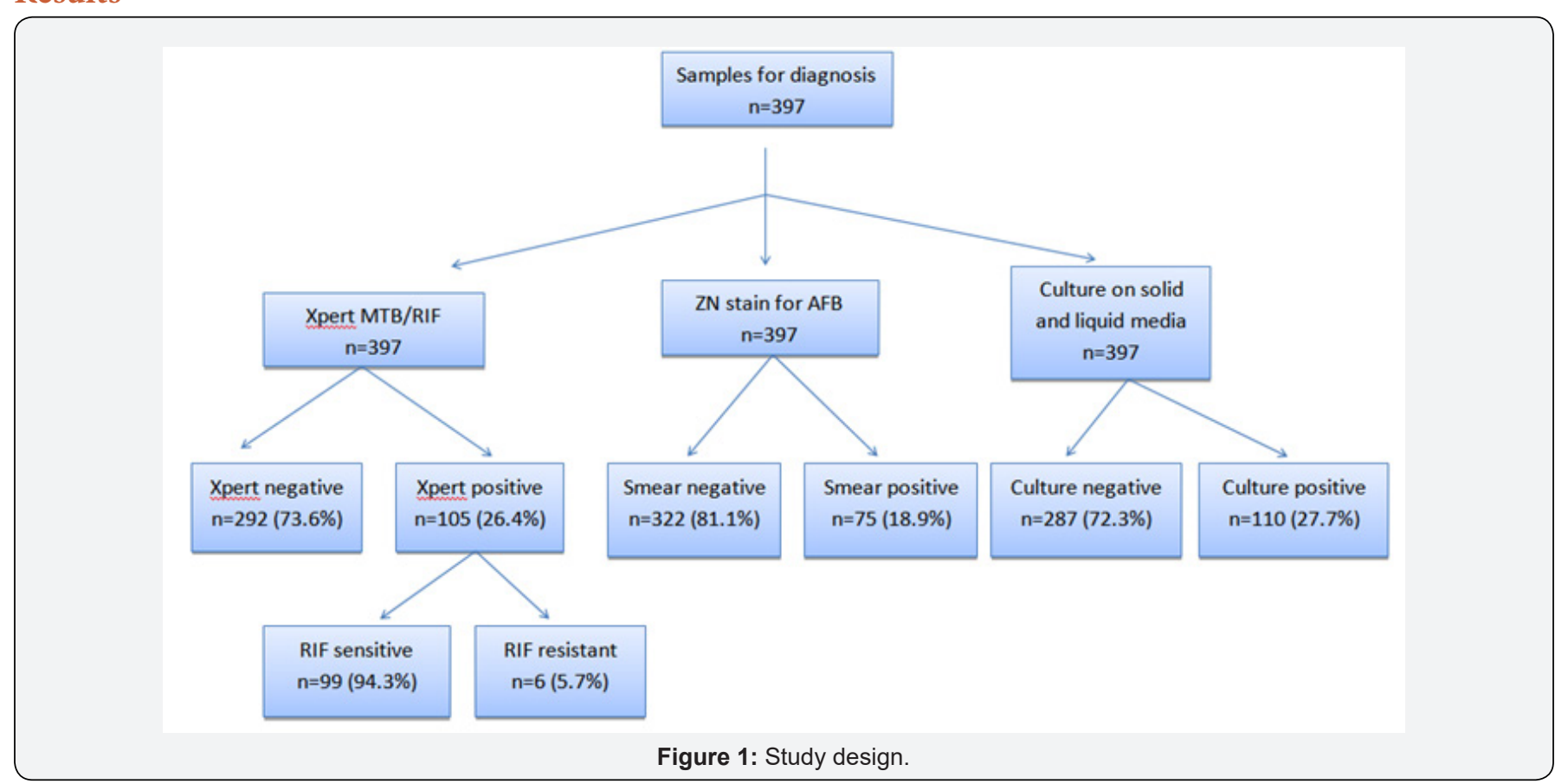


Total 397 patients were included in the study, youngest patient being 3 months and oldest being 11 years 5 month old. This Table 1 shows that majority of patients were males $(67.3 \%)$. Majority of patients belonged to the age group < 5 years (47.6\%). Only $18.4 \%$ of patients had history of contact with TB patient and $13 \%$ of patients showed positive Mantoux test (Table 2-4 \& Figure 1).

Table 1: Demographic profile of cases.

\begin{tabular}{|c|c|c|}
\hline Parameters & Number of cases & Percentage (\%) \\
\hline Gender & & \\
\hline Male & 267 & $67.30 \%$ \\
\hline Female & 130 & $32.70 \%$ \\
\hline Age group & & \\
\hline$<5$ years & 189 & $47.60 \%$ \\
\hline 5-9 years & 171 & $43 \%$ \\
\hline $10-12$ years & 37 & $9.40 \%$ \\
\hline $\begin{array}{c}\text { History of contact with } \\
\text { TB patient }\end{array}$ & 73 & $18.40 \%$ \\
\hline Yes & 324 & $81.60 \%$ \\
\hline No & 52 & $13 \%$ \\
\hline Mantoux test & 345 & $87 \%$ \\
\hline Positive & & \\
\hline Negative & & \\
\hline
\end{tabular}

Table 2: Type and number of samples used.

\begin{tabular}{|c|c|c|c|}
\hline \multirow{4}{*}{$\begin{array}{c}\text { Pulmonary } \\
(\mathrm{n}=325)\end{array}$} & $\begin{array}{c}\text { Type of sample } \\
\text { Sputum/Induced } \\
\text { sputum }\end{array}$ & $\begin{array}{c}\text { Total } \\
\text { samples }\end{array}$ & $\begin{array}{c}\text { MTB detected } \\
\text { by CBNAAT }\end{array}$ \\
\cline { 2 - 4 } & $\begin{array}{c}\text { Gastric lavage/ } \\
\text { aspirate }\end{array}$ & 224 & $74(33 \%)$ \\
\cline { 2 - 4 } & $\begin{array}{c}\text { Bronchoalveolar } \\
\text { lavage }\end{array}$ & 3 & $0(0 \%)$ \\
\hline \multirow{4}{*}{$\begin{array}{c}\text { Extrapulmonary } \\
\text { (n=72) }\end{array}$} & CSF & 55 & $7(12.7 \%)$ \\
\cline { 2 - 4 } & Aleural fluid & 10 & $1(10 \%)$ \\
\cline { 2 - 4 } & $\begin{array}{c}\text { FNAC material } \\
\text { from lymph node }\end{array}$ & 3 & $2(66.7 \%)$ \\
\hline & & 397 & 105 \\
\hline
\end{tabular}

Table 3: Performance of CBNAAT versus culture. CBNAAT detected MTB in 102 of the 110 culture positive cases(92.7\%) and in 3 of the culture negative cases( $1 \%)$.

\begin{tabular}{|c|c|c|c|}
\hline & Culture positive & Culturenegative & Total \\
\hline CBNAAT positive & 102 & 3 & 105 \\
\hline CBNAAT negative & 8 & 284 & 292 \\
\hline Total & 110 & 287 & 397 \\
\hline
\end{tabular}

Table 4: Performance of $\mathrm{ZN}$ smear versus culture. $\mathrm{ZN}$ smear detected MTB in 43 of the 110 culture positive cases(39.09\%) and in 32 of the culture negative cases(11.14\%).

\begin{tabular}{|c|c|c|c|}
\hline & $\begin{array}{c}\text { Culture } \\
\text { positive }\end{array}$ & $\begin{array}{c}\text { Culture } \\
\text { negative }\end{array}$ & Total \\
\hline $\begin{array}{c}\text { ZN smear } \\
\text { positive }\end{array}$ & 43 & 32 & 75 \\
\hline $\begin{array}{c}\text { ZN smear } \\
\text { negative }\end{array}$ & 67 & 255 & 322 \\
\hline Total & 110 & 287 & 397 \\
\hline
\end{tabular}

Table 5: Sensitivity, specificity, positive predictive value and negative predictive value of CBNAAT and ZN smear in reference to culture.

\begin{tabular}{|c|c|c|c|c|}
\hline & Sensitivity & Specificity & $\begin{array}{c}\text { Positive } \\
\text { predictive } \\
\text { value }\end{array}$ & $\begin{array}{c}\text { Negative } \\
\text { predictive } \\
\text { value }\end{array}$ \\
\hline $\begin{array}{c}\text { CBNAAT } \\
\text { versus } \\
\text { culture }\end{array}$ & $92.7 \%$ & $98.9 \%$ & $97.1 \%$ & $97.2 \%$ \\
\hline $\begin{array}{c}\text { ZN smear } \\
\text { versus } \\
\text { culture }\end{array}$ & $39.09 \%$ & $88.85 \%$ & $57.33 \%$ & $79.19 \%$ \\
\hline
\end{tabular}

Table 6: CBNAAT Performance in ZN smear positive and negative cases. CBNAAT detected MTB in 75 of the ZN smear positive cases and also in 30 of the $\mathrm{ZN}$ smear negative cases.

\begin{tabular}{|c|c|c|c|}
\hline & $\begin{array}{c}\text { ZN smear } \\
\text { positive for AFB }\end{array}$ & $\begin{array}{c}\text { ZN smear } \\
\text { negative for AFB }\end{array}$ & Total \\
\hline $\begin{array}{c}\text { MTB positive } \\
\text { by CBNAAT }\end{array}$ & 75 & 30 & 105 \\
\hline $\begin{array}{c}\text { MTB negative } \\
\text { by CBNAAT }\end{array}$ & 0 & 292 & 292 \\
\hline Total & 75 & 322 & 397 \\
\hline
\end{tabular}

Table 7: Sensitivity, specificity, positive predictive value and negative predictive value of CBNAAT in reference to $\mathrm{ZN}$ smear.

\begin{tabular}{|c|c|c|c|c|}
\hline & Sensitivity & Specificity & $\begin{array}{c}\text { Positive } \\
\text { predictive } \\
\text { value }\end{array}$ & $\begin{array}{c}\text { Negative } \\
\text { predictive } \\
\text { value }\end{array}$ \\
\hline $\begin{array}{c}\text { CBNAAT } \\
\text { versus ZN } \\
\text { smear }\end{array}$ & $100 \%$ & $90.68 \%$ & $71.42 \%$ & $100 \%$ \\
\hline
\end{tabular}

From the Tables 5-7 it is evident that sensitivity, specificity, positive predictive value and negative predictive value of CBNAAT are far better than ZN smear for diagnosing pediatric tuberculosis.

\section{Discussion}

The recent introduction of the CBNAAT assay has significantly revolutionized the diagnostics of tuberculosis in adults, but its application for the diagnosis of pediatric TB is under evaluation. To date, there are only a few studies on the application of CBNAAT for the diagnosis of pediatric tuberculosis in India, more so in eastern India. The Gene Xpert MTB/Rif test is a cartridge-based fully automated NAAT (nucleic acid amplification test) for TB case detection and rifampicin resistance testing, suitable for use in disease- 
endemic countries. It purifies, concentrates, amplifies (by rapid, real-time PCR) and identifies targeted nucleic acid sequences in the TB genome, and provides results from unprocessed sputum samples in less than 2 hours, with minimal hands-on technical time.

In the present study, 397 patients were included,youngest patient being 3 months and oldest being 11 years 5 month old, with male:female ratio 2.05:1.Majority of patients belonged to the age group $<5$ years $(47.6 \%)$. Only $18.4 \%$ of patients had history of contact with TB patient and 13\% of patients showed positive Mantoux test. Samples were taken from 325 pulmonary and 72 extrapulmonary TB patients. Among the pulmonary samples, CBNAAT detected MTB in 21 of the 98 sputum/induced sputum samples (21.4\%), 74 of the 224 gastric lavage/aspirate samples(33\%) and 0 of the 3 bronchoalveolar lavage samples(0\%). Among the extrapulmonary samples, CBNAAT detected MTB in 7 of the 55 CSF samples(12.7\%), 1 of the 10 pleural fluid samples(10\%), 0 of the 4 ascitic fluid samples(0\%) and 2 of the 3 lymph node aspirate samples obtained by FNAC(66.7\%).CBNAAT was positive in $105(26.4 \%)$ and was negative in 292 of the 397 cases(73.6\%). ZN smear detected AFB in 75 (18.9\%) and failed to detect AFB in 322 of the 397 cases (81.1\%). Culture was positive in $110(27.7 \%)$ and was negative in 287 of the 397 cases $(72.3 \%)$.

CBNAAT detected MTB in 102 of the 110 culture positive cases $(92.7 \%)$ and in 3 of the culture negative cases $(1 \%)$. So, there were 3 false positives who were CBNAAT positive but culture negative in our study. This was probably because 2 among these 3 patients had taken anti tubercular treatment for 4 months and then stopped treatment and 1 had stopped treatment after 3 months. CBNAAT can detect nucleic acids from dead and live organisms, so may remain positive long after treatment is completed and the culture is negative in these cases [18]. ZN smear detected MTB in 43 of the 110 culture positive cases (39.09\%) and in 32 of the culture negative cases $(11.14 \%)$. Sensitivity, specificity, positive predictive value and negative predictive value of CBNAAT in reference to culture are $92.7 \%, 98.9 \%, 97.1 \%$ and $97.2 \%$ respectively. Specificity was $98.9 \%$ with 8 false negatives who were CBNAAT negative but culture positive because 1 culture sample was positive for MOTT and CBNAAT only detects MTB. In other 7 samples, although MTB growth is in culture but it is possible that the bacterial load may have been too low for the CBNAAT to detect the DNA from MTB- complex. It shows that a patient with a negative CBNAAT can still have TB with MTB or MOTT [19]. Also, the negative predictive value is high as liquid culture method was used in our study in comparison to LJ media used in most of the earlier studies [19]. Sensitivity, specificity, positive predictive value and negative predictive value of ZN smear in reference to culture are 39.09\%, 88.85\%, $57.33 \%$ and $79.19 \%$ respectively. It is evident that sensitivity, specificity, positive predictive value and negative predictive value of CBNAAT are far better than ZN smear for diagnosing pediatric tuberculosis. CBNAAT detected MTB in 75 of the ZN smear positive cases and also in 30 of the $\mathrm{ZN}$ smear negative cases. Sensitivity, specificity, positive predictive value and negative predictive value of CBNAAT in reference to ZN smear are $100 \%, 90.68 \%, 71.42 \%$ and $100 \%$ respectively. CBNAAT also detected 6 cases of rifampicin resistance among the 105 cases detected by CBNAAT (5.7\%).

In the study of Sowjanya (Andhra Pradesh, India) (June 2012- December 2013), CBNAAT detected MTB in 144 out of 205 pulmonary specimens (70.24\% detection rate) whereas sputum for AFB was able to detect only 108 cases (52.68\% detection rate). CBNAAT detected 108 out of the 109 sputum smear positive cases, and 36 out of the 96 sputum smear negative cases [20]. In the study of Singh (Delhi, India), with culture as gold standard, sensitivity of CBNAAT was $84.6 \%$ and specificity was $86.4 \%$ [21]. Raizada tested 8,370 paediatric presumptive TB \& presumptive DR-TB cases between April-November 2014and showed that TB detection rates were two fold higher with CBNAAT as compared to smear microscopy [22]. The metaanalysis by Maynard-Smith showed that median sensitivity and specificity of Xpert assay with a culture based reference standard were 0.83 (IQR, 0.68-0.94) and 0.98 (IQR, 0.89-1.00) respectively [23]. Denkinger (USA) performed a systematic review and metaanalysis to assess the accuracy of Xpert for the detection of extrapulmonary TB. They determined the accuracy of Xpert compared with culture and a composite reference standard (CRS). They identified 18 studies involving 4461 samples. In lymph node tissues or aspirates, Xpert pooled sensitivity was $83.1 \%$ (95\% CI 71.4-90.7\%) versus culture and $81.2 \%$ (95\% CI 72.4-87.7\%) versus CRS. In cerebrospinal fluid, Xpert pooled sensitivity was $80.5 \%$ (95\% CI 59.0-92.2\%) against culture and62.8\% (95\% CI 47.7-75.8\%) against CRS. In pleural fluid, pooled sensitivity was $46.4 \%$ (95\% CI26.3$67.8 \%$ ) against culture and $21.4 \%$ (95\% CI 8.8-33.9\%) against CRS. Xpert pooled specificity was consistently $>98.7 \%$ against CRS across different sample types. Based on this systematic review, the World Health Organization now recommends Xpert over conventional tests for diagnosis of TB in lymph nodes and other tissues, and as the preferred initial test for diagnosis of TB meningitis [24]. Dewan (New Delhi, India) demonstrated that $10(25 \%)$ out of the 40 CBNAAT positive patients had rifampicin resistance. Among them 9 hadmultidrug resistant tuberculosis (MDR-TB) as detected by LPA [25]. Shah (Maharashtra, India) demonstrated that thesensitivity and specificity of Xpert MTB/RIF for bacteriologically (either AFB or culture positive) confirmed cases of tuberculosis were $80 \%$ and $71.4 \%$ [26]. An in-vitro study demonstrated a limit of detection as low as $131 \mathrm{CFU} / \mathrm{ml}$ of MTB by Xpert Assay [27]. Xpert will divert treatment away from "false cases" to "true" smear-negative TB cases, thereby increasing the accuracy of treatment and cost-effectiveness, while reducing the burdens 
of toxicity and cost of treatment in patients who do not in fact have TB. Sharma (New Delhi, India) described that Xpert has $77.7 \%$ sensitivity for smear negative and culture positive TB in comparison to $99.2 \%$ for smear and culture positive TB [28]. In the study of Mohanty (Cuttack, India)(April 2016 to March 2017), with culture as gold standard, sensitivity, specificity, positive predictive value(PPV) and negative predictive value(NPV) of sputum induction CBNAAT were $61.9 \%, 96.5 \%$, $96.3 \%, 63.6 \%$, respectively and of BAL CBNAAT were $88.09 \%$, 96.5\%, 97.36\%, 84.84\%, respectively [29]. Agrawal (Delhi, India, Jan 2015 to Nov 2015) demonstrated that using culture as reference, sensitivity, specificity, PPV and NPV of CBNAAT were $86.8 \%, 93.1 \%, 78.5 \%$ and $96 \%$ respectively [19].

Thus, our study adds data from TB endemic third world country that CBNAAT can help in rapid diagnosis of pediatric tuberculosis with high sensitivity, specificity, positive predictive value and negative predictive value. Also it simultaneously detects rifampicin resistance, so correct treatmentcan be started at an early stage of the disease.

It also detects true TB negative patients, thus, contributes to cost saving by avoiding unnecessary treatment. However the assay has severaldisadvantages:

1. Xpert cannot be used for assessing the emergence of rifampicin resistance during treatment. Also it is not suitable to detect isoniazid monoresistance.

2. Inability to differentiate extensively drug resistant (XDR-TB) from multi-drug resistant (MDR-TB) as it can detect only rifampicin resistance. It can detect rifampicin resistance only if rpoB allele is present in at least $65 \%$ of DNA present in sample.

3. It is not suitable for monitoring patients' response to treatment and so conventional microscopy and culture are required for monitoring MDR-TB patients during treatment.

4. Xpert Assay also have several technical problems, including requirement for stable electricity supply, limited temperature range, availability of maintenance, and bulky consumables.

Multicentric studies with larger sample size can be carried out to further validate the results, finding the positive and negative likelihood ratio, the cost effectiveness and patient acceptability. Likewise, studies on diagnostic usefulness of CBNAAT in diagnosing TB in patients infected with HIV will be useful because of the atypical clinical presentation of TB disease and the paucibacillary nature of pulmonary disease in patients with HIV. Also studies to compare rifampicin resistance found on Xpert MTB/RIF with that of drug susceptibility tests (DST) can be done.

\section{Conclusion}

CBNAAT assay is a rapid test which identifies both the presence of Mycobacterium tuberculosis (with high sensitivity, specificity, positive predictive value and negative predictive value) and resistance to rifampicin associated with mutation of rpoB gene in a single test. This can enable early and appropriate treatment initiation, as well as accelerating the implementation of MDR-TB control measures, and ultimately reducing TB case incidence. It also helps to avoid injudicious use of anti-tuberculosis drugs.Revised National TB Control Programme (RNTCP) is also currently using Xpert MTB/RIF to diagnose pulmonary TB, paediatric TB, extrapulmonary $\mathrm{TB}$ and rifampicin resistance and Multi Drug Resistance Tuberculosis in high risk populations like HIV positive as recommended by WHO under 2013 policy recommendations.

\section{References}

1. h t t p s : / / t b c i n d i a.g o v. i n / i n d e x 1 . php?lang=1\&level $=2 \&$ sublinkid $=4569 \&$ lid $=3174$

2. Marais B, Hesseling A, Gie R, Schaaf H, Beyers N (2006) The burden of childhood tuberculosis and the accuracy of community-based surveillance data. Int J Tuberc Lung Dis 10(3): 259-263.

3. Kumar A, Gupta D, Nagaraja S, Singh V, Sethi GR, et al. (2013) Updated National Guidelines for Pediatric Tuberculosis in India, 2012. Indian Pediatr 50: 301-306.

4. WHO (2013) Global Tuberculosis report 2013. World Health Organization, Geneva, Switzerland.

5. Revised National Tuberculosis Control Program (2017) TB India 2015, RNTCP Annual Status Report, Reach The Unreached, 2015. TB India, India.

6. Rachow A, Clowes P, Saathoff E, Mtafya B, Michael E, et al. (2012) Increased and expedited case detection by Xpert MTB/RIF assay in childhood tuberculosis: a prospective cohort study. Clin Infect Dis 54(10): 1388-1396.

7. Enarson PM, Enarson DA, Gie R (2005) Management of tuberculosis in children in low-income countries. Int J Tuberc Lung Dis 9(12): 12991304.

8. Raj A, Singh N, Mehta PK (2014) Gene Xpert MTB/RIF Assay: A New Hope for Extra-pulmonary Tuberculosis. IOSR J Pharm Biol Sci 2(1): 083-089.

9. Haldar S, Bose M, Chakrabarti P, Daginawala HF, Harinath BC, et al. (2011) Improved laboratory diagnosis of tuberculosis--The Indian experience. Tuberculosis (Edinb) 91(5): 414-426.

10. Ichhpujani RL, Agarwal SP, Chauhan LS (2005) Diagnostic needs and status of new diagnostic tools for tuberculosis. In: Agarwal SP, Chauhan LS.Tuberculosis control in India. Directorate General of Health Services, Ministry of Health and Family Welfare, Government of India, New Delhi, India, pp. 165-178.

11. Bianchi L, Galli L, Moriondo M, Veneruso G, Becciolini L, et al. (2009) Interferon-gamma release assay improves the diagnosis of tuberculosis in children. Pediatr Infect Dis J 28(6): 510-514.

12. Pai M, Flores LL, Pai N, Hubbard A, Riley LW, et al. (2004) Nucleic acid amplification tests in the diagnosis of tuberculous pleuritis: a systematic review and meta-analysis. BMC Infect Dis 4: 6 .

13. Dinnes J, Deeks J, Kunst H, Gibson A, Cummins E, et al. (2007) A systematic review of rapid diagnostic tests for the detection of tuberculosis infection. Health Technol Assess 11(3): 1-196.

14. Pai M, Nathavitharana R (2014) Extrapulmonary Tuberculosis: New Diagnostics and New Policies. Indian J Chest Dis Allied Sci 56(2): 71-73. 
15. World Health Organization (2017) Automated real-time nucleic acid amplification technology for rapid and simultaneous detection of tuberculosis and rifampicin resistance: Xpert MTB/RIF assay for the diagnosis of pulmonary and extrapulmonary TB in adults and children: policy update. World Health Organization, Geneva, Switzerland.

16. http://www.who.int/tb/publications/xpert_implem_manual/en/

17. https://tbcindia.gov.in/WriteReadData/TB\%20India\%202017.pdf

18. Barnard DA, Irusen EM, Bruwer JW, Plekker D, Whitelaw AC, et al (2015) The utility of Xpert MTB/RIF performed on bronchial washings obtained in patients with suspected pulmonary tuberculosis in a high prevalence setting. BMC Pulm Med 15: 103.

19. Agrawal M, Bajaj A, Bhatia V, Dutt S (2016) Comparative Study of GeneXpert with ZN Stain and Culture in Samples of Suspected Pulmonary Tuberculosis. J Clin Diagn Res 10(5): DC09-DC12.

20. Sowjanya DS, Behera G, Reddy VVR, Praveen JV (2014) CBNAAT: a Novel diagnostic tool for rapid and specific detection of Mycobacterium Tuberculosis in pulmonary samples. Int J Res Med Sci 1(1): 28-31.

21. Singh M, Sethi GR, Mantan M, Khanna A, Hanif M (2016) Cartridge Based Nucleic Acid Amplification Test (CBNAAT) For The Diagnosis Of Pulmonary Tuberculosis In Children. American Journal of Respiratory and Critical Care Medicine 193: A7695.

22. Raizada N, Sachdeva KS, Swaminathan S, Kulsange S, Khaparde SD, et al. (2015) Piloting Upfront Xpert MTB/RIF Testing on Various Specimens under Programmatic Conditions for Diagnosis of TB \& DR-TB in Paediatric Population. PLoS One 10(10): e0140375.
23. Maynard-Smith L, Larke N, Peters JA, Lawn SD (2014) Diagnostic accuracy of the Xpert MTB/RIF assay for extrapulmonary and pulmonary tuberculosis when testing non-respiratory samples: a systematic review. BMC Infect Dis 14: 709.

24. Denkinger CM, Schumacher SG, Boehme CC, Dendukuri N, Pai M, et al. (2014) Xpert MTB/RIF assay for the diagnosis of extrapulmonary tuberculosis: asystematic review and meta-analysis. Eur Respir J 44(2): 435-446.

25. Dewan R, Anuradha S, Khanna A, Uppal S, et al. (2015) Role of cartridgebased nucleic acid amplification test (CBNAAT) for early diagnosis of pulmonary tuberculosis in HIV. Journal of Indian Academy of Clinical Medicine 16(2): 114-117.

26. Shah I, Gupta Y (2016) Xpert MTB/RIF for Diagnosis of Tuberculosis and Drug Resistance in Indian Children. Indian Pediatr 53(9): 837-838.

27. http://www.who.int/tb/laboratory/xpert_faqs.pdf

28. Sharma SK, Kohli M, Yadav RN, Chaubey J, Bhasin D, et al. (2015) Evaluating the Diagnostic Accuracy of Xpert MTB/RIF Assay in Pulmonary Tuberculosis. PLoS One 10(10): e0141011.

29. Mohanty T, Panigrahi SK, Pattnaik M, Panda G, Routray D, et al. (2017) Study on diagnostic modalitiesin smear negative pulmonary tuberculosis with special reference to sputum induction (SI CBNAAT), bronchoscopy (BAL CBNAAT and BAL culture). J Evid Based Med Healthc 4(47): 2858-2862.

\section{Your next submission with Juniper Publishers will reach you the below assets}

- Quality Editorial service

- Swift Peer Review

- Reprints availability

- E-prints Service

- Manuscript Podcast for convenient understanding

- Global attainment for your research

- Manuscript accessibility in different formats

( Pdf, E-pub, Full Text, Audio)

- Unceasing customer service

Track the below URL for one-step submission https://juniperpublishers.com/online-submission.php 